IV.

\title{
5 Schläfebeine mit Brüchen
}

Klinisohe und pathologische Mitteilungen IX.

Mit 12 Abbildungen nach Zeichnungen des Verfassers

ron

Rudolf Panse in Dresden-Neustadt.

1. 30jähriger Lehrer C. M. 8. Juni 03 auf dem Rade mit dem Gesicht gegen einen Baum gefahren. 1/2 Stunde bewubtlos, einige Tage drehte sich die Decke nach rechts. Blut kam aus Nase und Ohr.

29. Juni. Trommelfell undeutlieh rot, rauher Knochen vorn unten im Gehörgang sichtbar.

$$
\begin{aligned}
& \text { Hörprüfung } \\
& \mathrm{R}^{\mathrm{cS}} \mathrm{L} \\
& \text { Diagnose Gehörgangsbruch } \\
& \text { durch das Kieferköptchen } \\
& \begin{array}{r}
-3 c^{4} L-3 \\
+\frac{c L}{4}-4 \\
4+c W+9
\end{array} \\
& \text { Fl } 624 \mathrm{~m} \\
& \mathrm{St} \\
& \begin{array}{cc}
C^{2} \mathrm{C}, \mathrm{Cc} & \mathrm{C}, \mathrm{Cc} \\
c^{1} c^{2} c^{3} c^{4} & \mathrm{c}^{1} \mathrm{c}^{2} \mathrm{c}^{3} c^{4} \\
c^{5} c^{6} c^{7} c^{8} & c^{5} c^{6} c^{7} c^{8}
\end{array}
\end{aligned}
$$

Operation am 14. Juli. Sehr stark blutende Weichteile, versehiedene abnorme Bänder, sodal die Gehörgangshaut sehwer

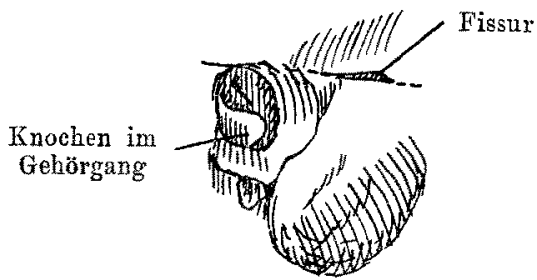

Fig. 1.

abzusehieben ist. Aus der fissura tympanomastoide starke Blutung. Gehörgang knöchern, etwas federnd, von unten verengt; 


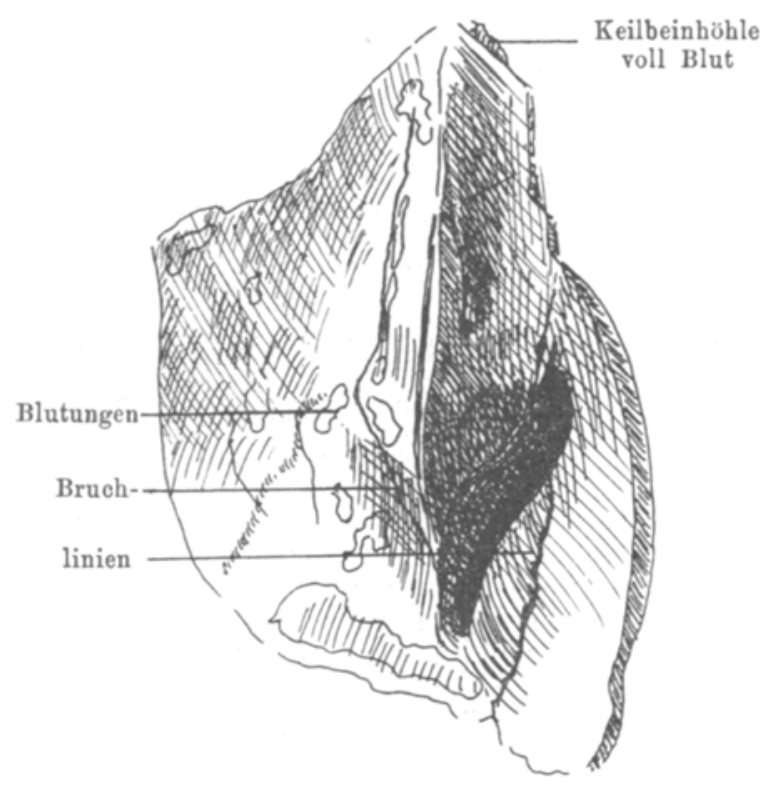

Fig. 2.

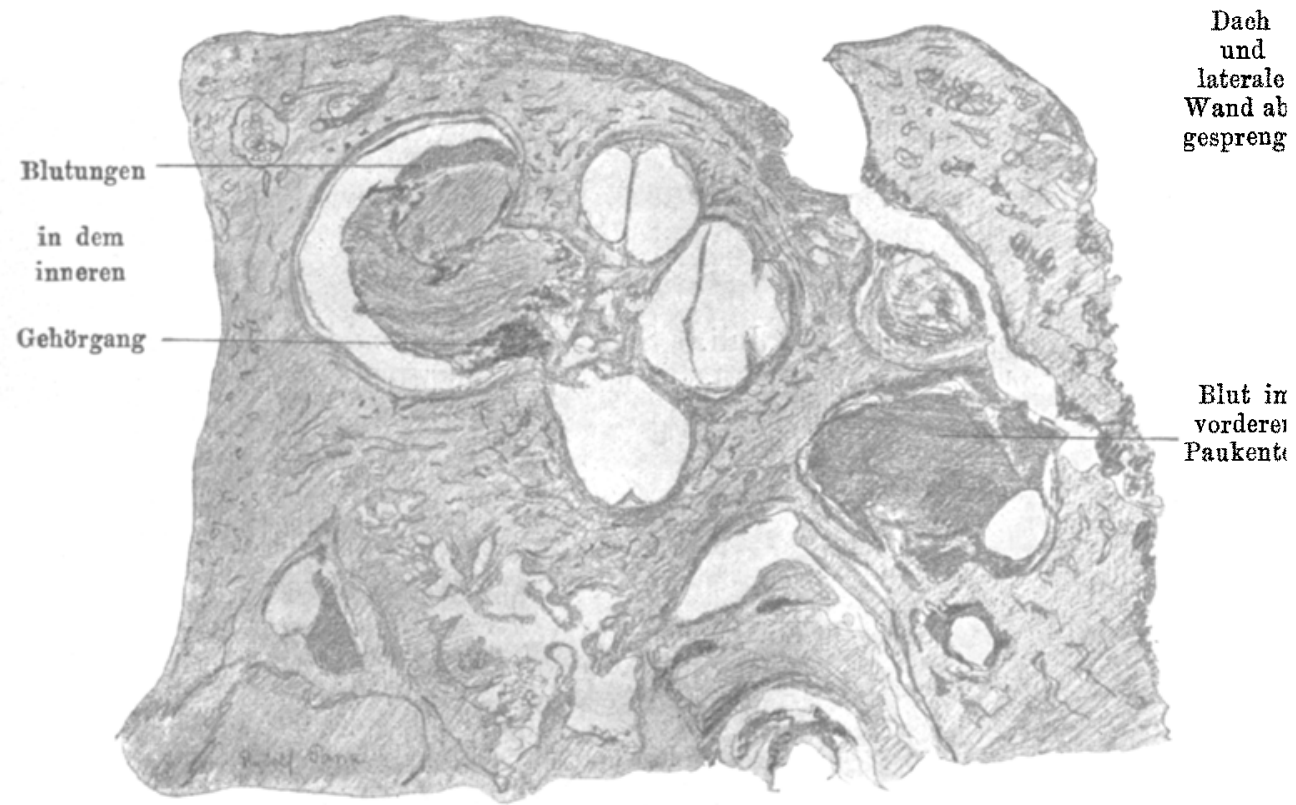

Fig. 3. 


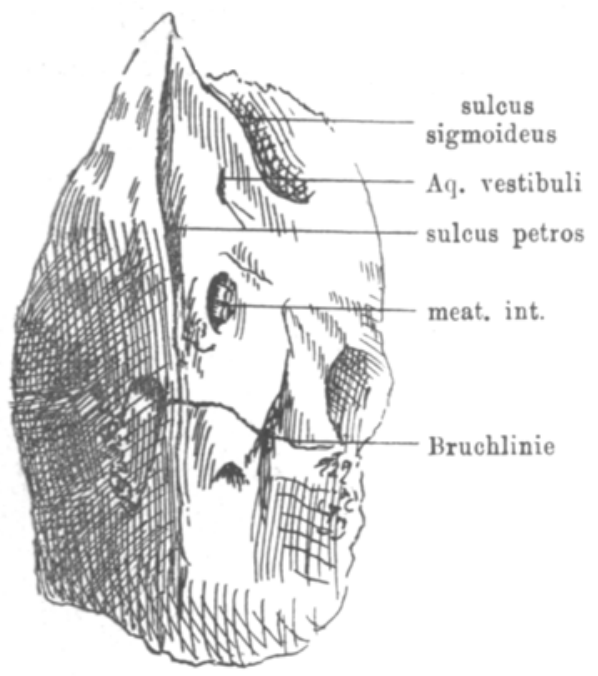

Fig. 4.

ein $5 \times 3 \mathrm{~mm}$ großes Knochenstick abgemeißelt. Der ganze Warzenfortsatz vorn unten blauroth, voll Blut; oben eine Fissur.

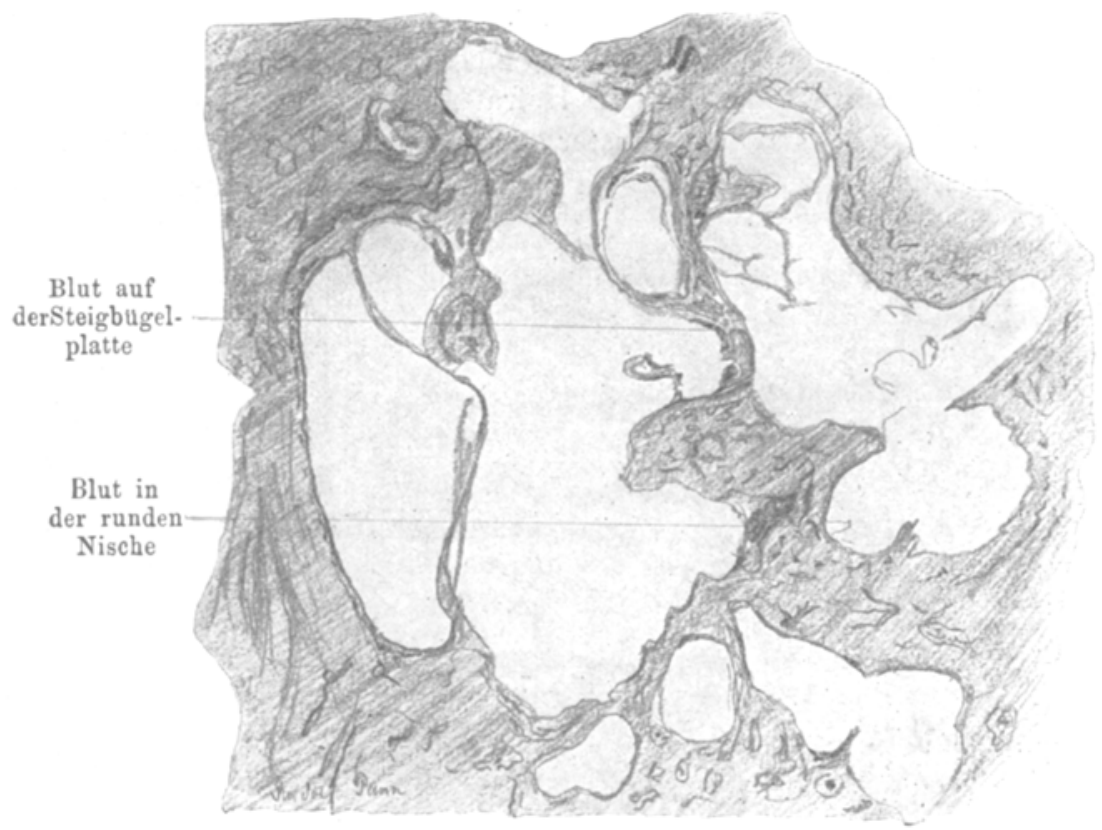

Fig 5 . 
(Siehe Abb. 1.) Unten einige Nähte. Nach 11 Tagen ans der Klinik entlassen. Heilung ohne Besonderes.

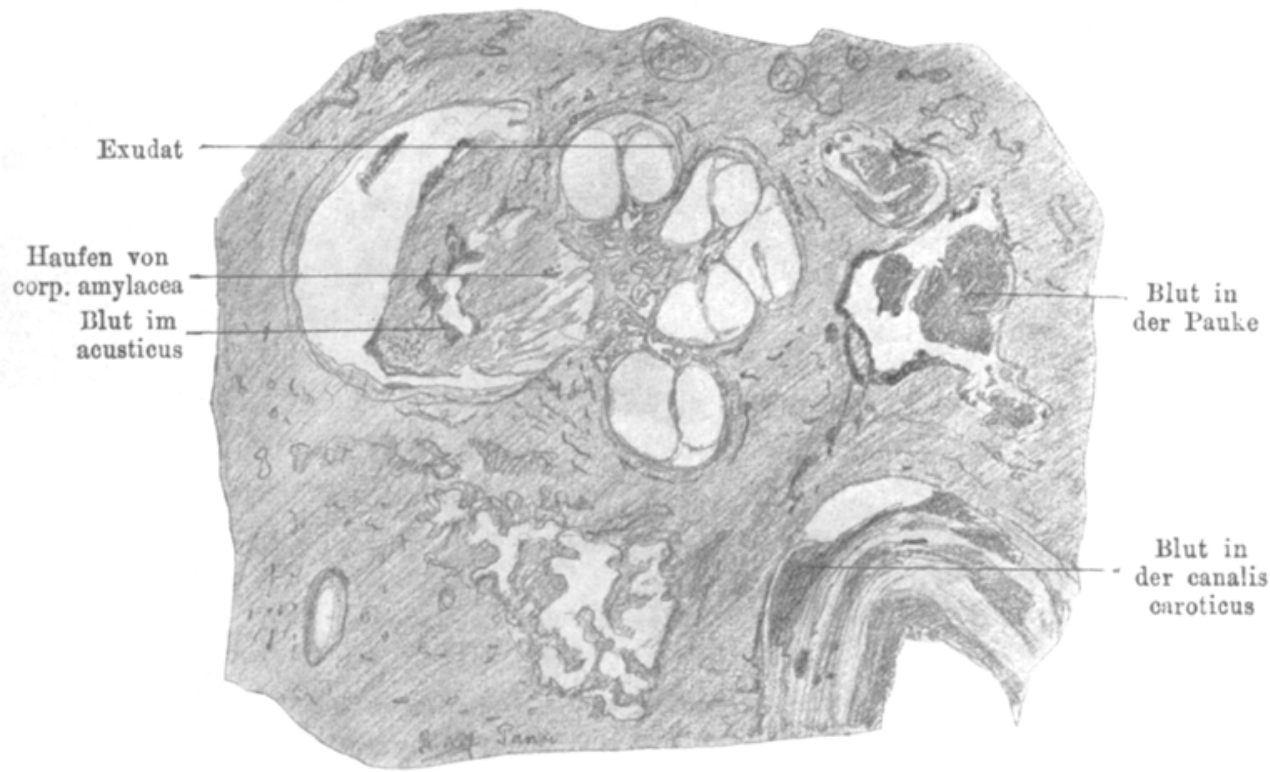

Fig. 6.

2. Geschenk des Herrn Hofrat Dr. Ganser. Von einem Irren, der sich aus dem Fenster stürzte. Es haben sich 2 Längsfissuren durch das Schläfenbein gebildet, eine durch das Tegmen

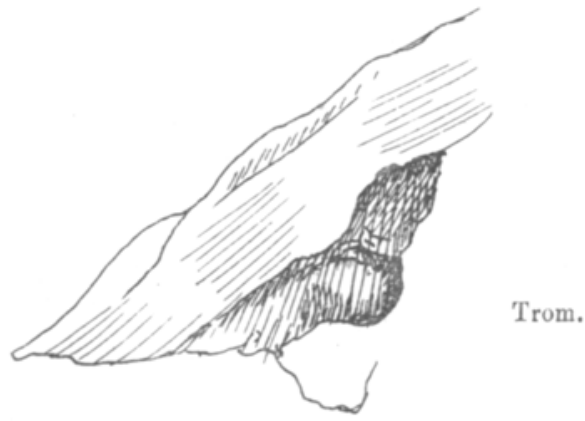

Fig. 7 .

Tympani, eine durch den sulcus sigmoideus schief verlaufend. (Siehe Abb. 2.) Mikroskopisch Blut um den acusticus im inneren Gehörgang und in der Pauke. (Abb. 3.) 


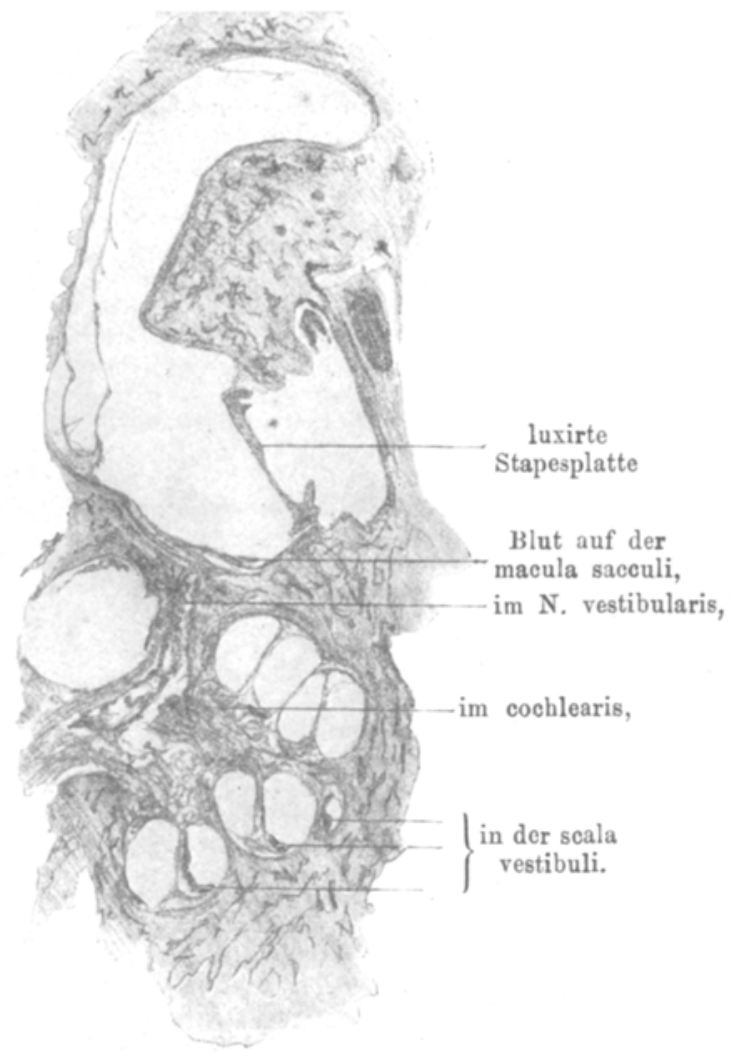

Fig. 8. Horizontalschnitt.

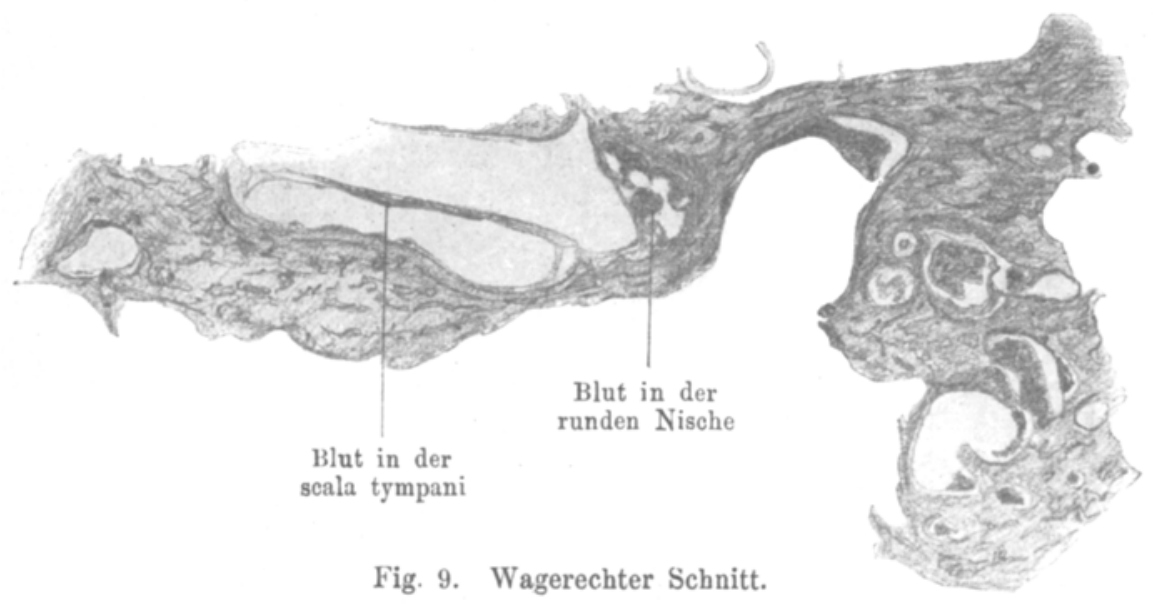


3. Gesehenk von Herrn Geh. Medizinalrat Prof. Dr. Schmorl. Querbruch durch das rechte Sehläfenbein. (Abb. 4.) In der Panke

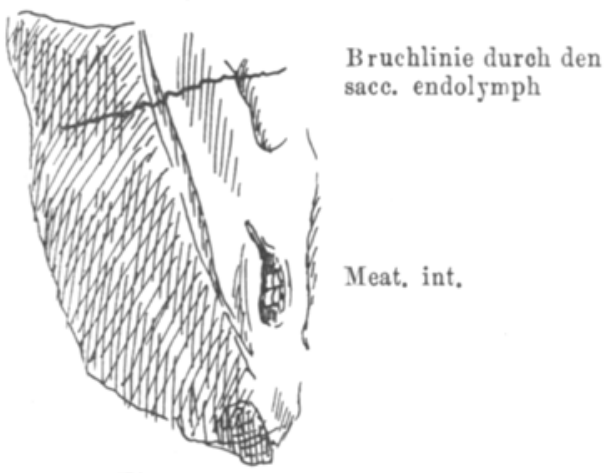

Fig. 10.

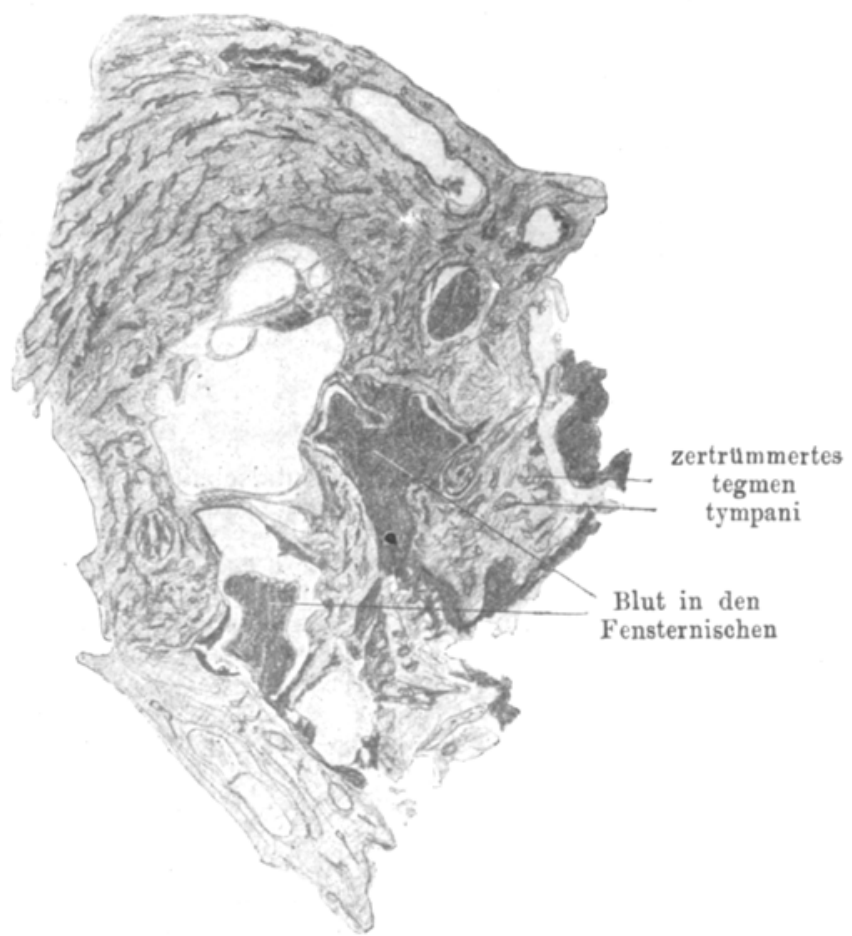

Fig. 11 .

nur in den Nischen geringe Blutungen (Abb. 5) und nach der Tube zu etwas mehr. Auberdem im canalis caroticus und 
zwischen den Hörnervenfasern. (Abb. 6.) Ich glaubte erst die auberordentlich zahlreichen corpora amylacea als Zerfallsprodukte des durch die Blutung zerstörten Nerven auffassen zu müssen, da sie aber bei den anderen Schläfenbeinen fehlten ist der Befund wohl zufällig.

4. Geschenk von Herrn Prosector Dr. Oppe. Linkes Schläfenbein, der Felsenteil ist vom Schuppenteil durch einen Längsbruch

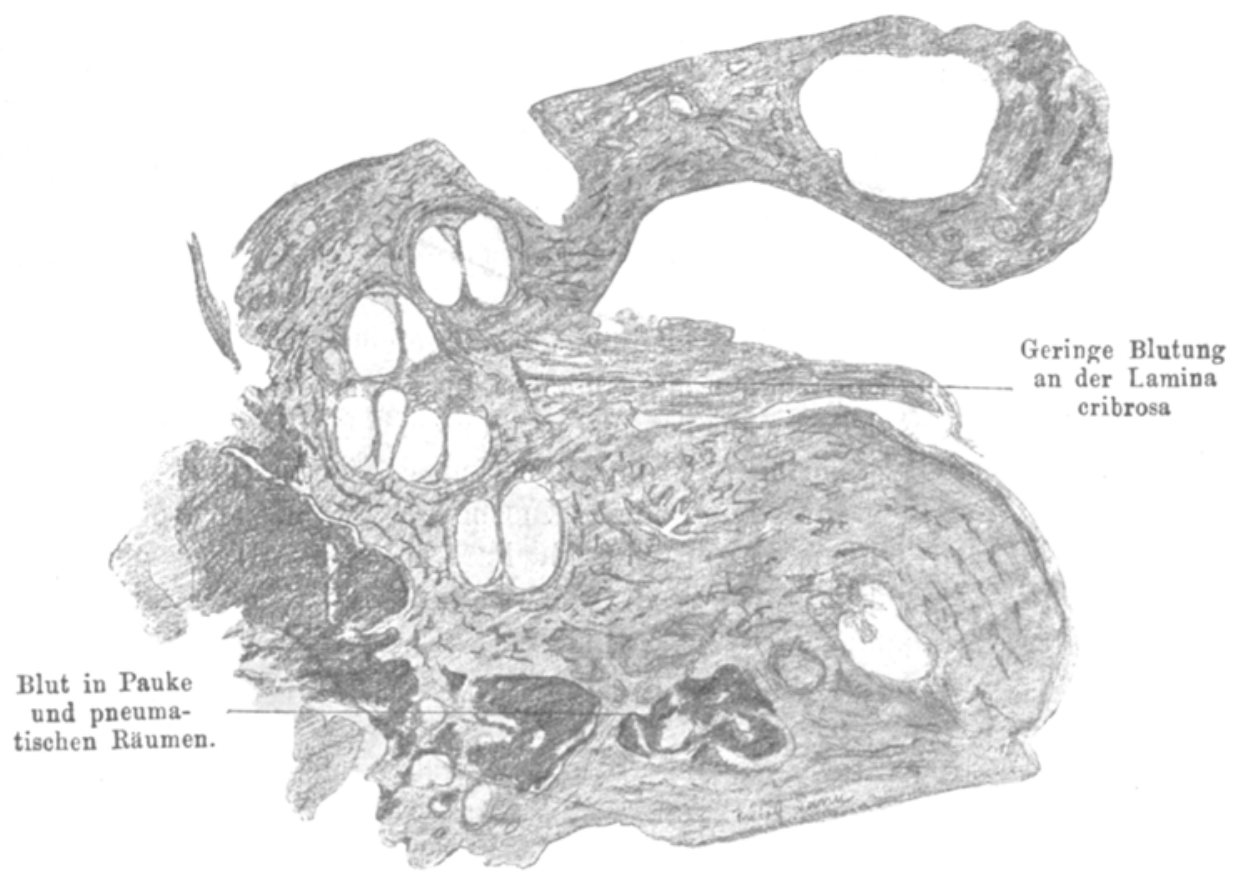

Fig. 12.

abgetrennt. (Abb. 7.) Die Horizontalsehnitte zeigen (Abb. 8) eine Luxation des Steigbïgels nacb innen, Blut auf der macula sacculi, im Nervus vestibularis und cochlearis und in der scala vestibuli in den nach vorn gelegenen Teilen, in einem tiefern Schnitt Blut in der runden Nische unả in den Luftzellen. (Abb. 9.)

Rechts ging eine quere Bruchlinie durch den sacculus endolmphaticus. (Abb. 10.) Pauke und Fenster ganz mit Blut angefüllt. (Abb. 11.) Vorhof und Schnecke frei, an der lamina cribrosa kleine Blutungen. 